\title{
A Method for Visualizing Independent Spatio-Temporal Patterns of Brain Activity
}

\author{
Dean J. Krusienski \\ School of Engineering, University of North Florida, 1 UNF Drive, Jacksonville, FL 32224, USA \\ Correspondence should be addressed to Dean J. Krusienski, deankrusienski@ieee.org
}

Received 2 January 2009; Accepted 31 May 2009

Recommended by Don Johnson

\begin{abstract}
Evoked and coordinated brain signals often exhibit distinct, individualized spatial and temporal characteristics, such as amplitude and phase couplings across and within spatial channels. In the study of these brain potentials, it is important to characterize both the spatial and temporal morphologies of the responses for a better understanding of both the physiology and function of the brain. This paper presents a method for visualizing the characteristic spatio-temporal brain activity associated with two distinct conditions. This method, called Independent Spatio-Temporal Patterns (ISTPs), extends Common Spatial Patterns (CSPs) for spatio-temporal pattern visualization by adding temporal features. Independent component analysis (ICA) is then applied to extract independent spatio-temporal patterns corresponding to each condition. The results indicate that the inclusion of temporal features can provide useful insight regarding the spatio-temporal characteristics of sensorimotor rhythms.
\end{abstract}

Copyright (๑) 2009 Dean J. Krusienski. This is an open access article distributed under the Creative Commons Attribution License, which permits unrestricted use, distribution, and reproduction in any medium, provided the original work is properly cited.

\section{Introduction}

Evoked and coordinated brain signals often exhibit distinct, individualized spatial and temporal characteristics, such as amplitude and phase couplings across and within spatial channels [2]. In the study of these brain potentials, it is important to characterize both the spatial and temporal morphologies of the responses for a better understanding of both the physiology and function of the brain. This knowledge is valuable for characterizing new responses, mapping brain activity, detecting irregularities, and controlling a braincomputer interface (BCI).

Simple ensemble averaging is usually sufficient to characterize stimulus evoked potentials because the phase of the response is typically time-locked to the stimulus [2]. However, the averaging of event related (de)synchronizations, such as sensorimotor rhythms (SMRs), is more difficult because the phase of the responses is not time locked to a stimulus. Although the general spatial and spectral characteristics of SMRs are well characterized, there can be differences ranging from subtle to significant in the spatio-temporal SMR patterns across individuals [4]. Obtaining a characteristic temporal waveform from (de)synchronizations on a single channel can be straightforwardly accomplished via averaging using correlations of known responses [4]. However, this method is not precise for examining multiple channels simultaneously. The proposed method, Independent SpatioTemporal Patterns (ISTPs), extends common spatial patterns (CSPs), combined with independent component analysis (ICA) in order to accurately extract such characteristic spatio-temporal patterns.

The method of common spatial patterns (CSPs) $[5,6]$ determines an optimal set of spatial filters for discriminating between two classes. It has been extended to multiclass paradigms [7] and proved successful for visualizing and classifying different mental states for brain-computer interfaces (BCIs) [3, 8-11]. However, CSP does not consider the short-time temporal characteristics of the data, such as the phase relationships between channels and frequency bands. The methods of common Spatio-spectral patterns (CSSPs) [1] and common sparse spectral spatial patterns (CSSSPs) [21] extend the spatial filtering approach to include time delay embedding in order to create a more flexible spatial-spectral filter. The components obtained by these time-embedded methods are not necessarily independent, and, because the filtering matrices are temporally sparse, it is not as straightforward to extract the representative spatio-temporal patterns for visualization. The method of Independent Spatio-Temporal Patterns (ISTPs) presented 
here also utilizes time delay embedding, with the application of independent component analysis (ICA) to the resulting patterns. In this case, uniform temporal sampling is used to construct the filtering matrix, enabling a complete visualization of the discriminable spatio-temporal patterns for the two classes. The addition of ICA produces a set of independent spatio-temporal patterns that are informative in terms of substantiating the morphology of the EEG during different mental states.

\section{The Method of Common Spatial Patterns}

The CSP decomposition of a feature matrix is given as,

$$
Y=W X
$$

where $X$ is an $\mathrm{N}$ feature $\times \mathrm{T}$ observance matrix, $W$ is an $\mathrm{L}$ $\times \mathrm{N}$ matrix $(\mathrm{L} \leq \mathrm{N})$ whose $\mathrm{L}$ rows represent the individual components of the decomposition, and $Y$ is an $\mathrm{L} \times \mathrm{T}$ matrix subspace of $X$. For a two-class classification problem, $W$ can be determined to decompose the feature matrix such that the resulting projections corresponding to the extreme eigenvalues of the transformed covariance matrices have maximal variance for one class and minimal variance for the other class. First, for the two classes (1 and 2), the classlabeled observations are sorted by the respective class and the class-specific covariance matrices are determined:

$$
\Sigma_{1}=X_{(1)} X_{(1)}^{T}, \quad \Sigma_{2}=X_{(2)} X_{(2)}^{T} .
$$

The task is defined as finding the transformation $W$ to create projections that simultaneously maximize the variance for one class and minimize the variance for the other:

$$
W \Sigma_{1} W^{T}=D, \quad W \Sigma_{2} W^{T}=I-D,
$$

where $D$ is a diagonal matrix with elements in $[0,1]$. This can be accomplished through simultaneous diagonalization of the two covariance matrices. First, a whitening transformation is performed:

$$
P\left(\Sigma_{1}+\Sigma_{2}\right) P^{T}=I
$$

Using spectral theory, the eigenvalue decomposition is then performed for the transformed classes:

$$
P \Sigma_{1} P^{T}=R D R^{T}, \quad P \Sigma_{2} P^{T}=R(I-D) R^{T},
$$

where the columns of $R$ are the eigenvectors, and the diagonal elements of $D$ and $(D-I)$ are the eigenvalues of classes 1 and 2, respectively. Note that the maximum eigenvalues for one class correspond to the minimum eigenvalues for the other class. By selecting only the eigenvectors corresponding to the largest and smallest eigenvalues that provide the best discrimination between classes, the subspace projection matrix is defined as.

$$
\widetilde{W}=\widetilde{R}^{T} P .
$$

For standard CSP analysis of EEG, the features of $X$ are simply the instantaneous bandpass-filtered voltages at each electrode. For ISTP, the features are the concatenation of time-windowed voltages for each electrode. The actual EEG patterns corresponding to the two mental states can be visualized by inverting the filtering matrix $W$.

\section{Methodology}

In one- and two-dimensional BCI cursor control studies [13$16]$, trained users are able to effectively modulate $8-12 \mathrm{~Hz}$ ( $\alpha$ band) and $18-26 \mathrm{~Hz}$ ( $\beta$ band) spectral components over the sensorimotor cortex to move a cursor toward a randomly positioned target on a monitor. Four sessions of data from ten able-bodied users (six women and four men ranging in age from 29 to 45 ) who performed a one-dimensional twotarget cursor control task were used for offline evaluation of the ISTPs. All users had exhibited strong $\mu$-band activity during an initial screening and were subsequently trained on a simple two-target, one-dimensional cursor control task. All users were successfully trained on the task (consistently $>80 \%$ accuracy) and ranged in experience from 1 to 20 sessions on the task prior to this data set. The study was approved by the New York State Department of Health Institutional Review Board, and each user gave informed consent.

\subsection{One-Dimensional Sensorimotor Rhythm Cursor Control Task. The one-dimensional sensorimotor rhythm cursor control task is shown in Figure 1. For the task, the users were presented by a target randomly positioned at the top or bottom right edge of the monitor. The trial began with the cursor at the left center of the monitor. It moved at a constant rate toward the right, reaching the right side of the monitor after 2 seconds. The users' goal was to move the cursor upward or downward to the height of the target so that it hits the target when it reached the right side of the monitor. The trials continued in 3-minute runs, with a 1-minute break given between runs. Between 18 and 30 trials were completed in a single 3-minute run, and 8 runs constitute a single session. Sessions were conducted one per day over a period of several weeks.}

3.2. Data Collection and Feature Extraction. The details of the data collection and analysis are as follows. Using BCI2000 software [17], the EEG activity was collected from 64 channels at standard locations [18] distributed over the scalp. All 64 channels were referenced to the right ear, bandpass filtered $(0.1-60 \mathrm{~Hz})$, and digitized at $160 \mathrm{~Hz}$. For each user, a large Laplacian spatial filter [12] (see Figure 2) was applied to the electrode over the right-or-left hand area of the sensorimotor cortex that exhibited maximal correlation between $\mu$-band activity and the task based on prior sessions. A $3-\mathrm{Hz}$ bin at the user-specific $\mu$-band frequency from a 16th-order AR model was extracted from the spatialfiltered signal and used as the online control feature. The AR feature was calculated every 50 milliseconds from the past 400 milliseconds of data. The AR model order, bin width, and time windowing were selected based on extensive empirical evaluations. The specific locations and frequencies used for the online experiments are provided in Table 1.

\section{Offline Analysis}

The data set used for offline analysis, collected as described in Section 3.2, consisted of 4 sessions of 8 runs each from 


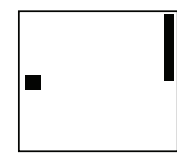

1

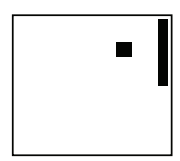

2

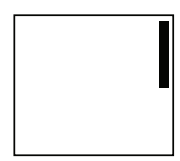

3

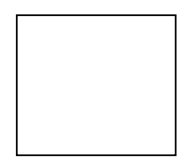

4

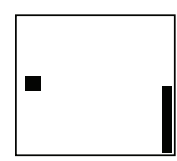

5

Figure 1: One-dimensional task trial structure. (1) The target and cursor are present on the screen for 1 second. (2) The cursor moves steadily across the screen for 2 seconds with its vertical movement controlled by the user. (3) If the user hits the target, the target flashes for 1.5 seconds. If the cursor misses the target, the screen is blank for 1.5 seconds. (4) The screen goes blank for a 1-second interval. (5) The next trial begins.

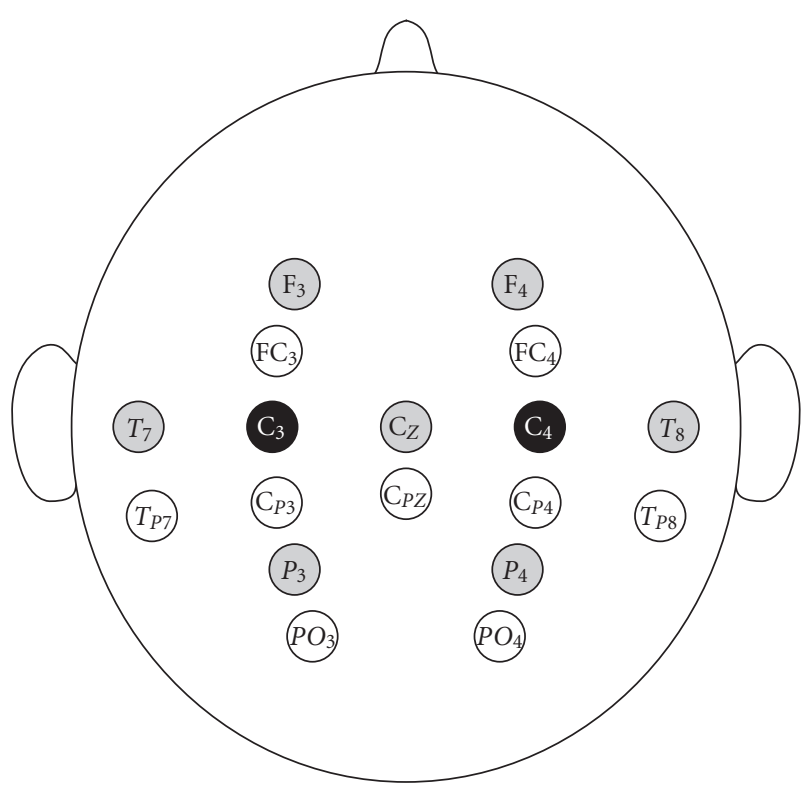

Figure 2: The large Laplacian spatial filter configuration. For an electrode over the hand area of the sensorimotor cortex (indicated in black), the signals from the four equidistant surrounding electrodes (indicated in gray) are averaged and subtracted from this central (black) electrode to produce the control signal. For the online experiments, a single large Laplacian control signal was used for cursor control. For the offline analysis, the 9 electrodes comprising the two contralateral Laplacian filters covering both hemispheres (depicted as the shaded locations for the $\mathrm{C}_{3} / \mathrm{C}_{4}$ users and as the unshaded locations for the $C_{P 3} / C_{P 4}$ users) were used for processing.

each of the 10 users. The first session was used to derive the ISTPs, and the three subsequent sessions were used for validation. As a necessary preprocessing step for CSP-based methods, the channels were bandpass filtered from 7 to $30 \mathrm{~Hz}$ [1]. The spatio-temporal features were constructed using the nine raw (i.e., ear-referenced) channels that represent the union of the channels that comprise large Laplacians over the left and right sensorimotor cortex. The observations used to construct the covariance matrices were collected every 6.25 milliseconds using the past 400 milliseconds of data, thus matching the segment length used for the online experiments.

The observations were collected every 6.25 milliseconds to provide an ample number of observations for constructing the covariance matrices, although a lower observation rate
TABLe 1: The predetermined optimal electrode location (International 10-20 System [18]) and fundamental $\mu$-rhythm frequency for each user.

\begin{tabular}{lcc}
\hline User & Location & Frequency $(\mathrm{Hz})$ \\
\hline $\mathrm{A}$ & $C_{3}$ & 12 \\
$\mathrm{~B}$ & $C_{3}$ & 12 \\
$\mathrm{C}$ & $C_{P 3}$ & 12 \\
$\mathrm{D}$ & $C_{3}$ & 12 \\
$\mathrm{E}$ & $C_{P 3}$ & 11 \\
$\mathrm{~F}$ & $C_{3}$ & 12 \\
$\mathrm{G}$ & $C_{4}$ & 12 \\
$\mathrm{H}$ & $C_{P 4}$ & 13 \\
$\mathrm{I}$ & $C_{3}$ & 12 \\
$\mathrm{~J}$ & $C_{3}$ & 10 \\
\hline
\end{tabular}

would likely be adequate. The channel set was limited to nine electrodes in order to provide comparison to the Laplacian spatial filter as well as a manageable feature space for the ISTP method.

4.1. Common Spatio-Temporal Patterns. Prior to performing the ICA, the Common Spatio-Temporal Patterns (CSTPs) can be visualized by inverting the projection matrix $W$. Interestingly, for all users, the components representing the two most extreme eigenvalues for the target corresponding to SMR synchronization (high SMR-band amplitude) were nearly identical 90 degree phase-shifted sinusoidal waveshapes at the $\mu$-band control frequency with maximal amplitude over the electrode used for control as illustrated in Figure 3 (notice that the relative amplitudes of the different channels are not equally proportional to the distance from the central electrode as approximated by a Laplacian spatial filter). Based on Fourier theory, a linear combination of these two components can model a sinusoidal response with arbitrary phase. This decomposition is logical since the phase of the EEG is not time-locked to the observation intervals. Additionally, for the majority of users, two of the remaining four most prominent components for the synchronized condition are sinusoids in the $\beta$ band having similar topographies and proportionally lower amplitudes to the $\mu$-band components (see Figure 3). It is worth noting that the precise frequencies of the SMR activity can be determined from the periods of the corresponding CSTPs, which are potentially more accurate than estimates obtained from a standard spectral decomposition. For the desynchronized condition, 


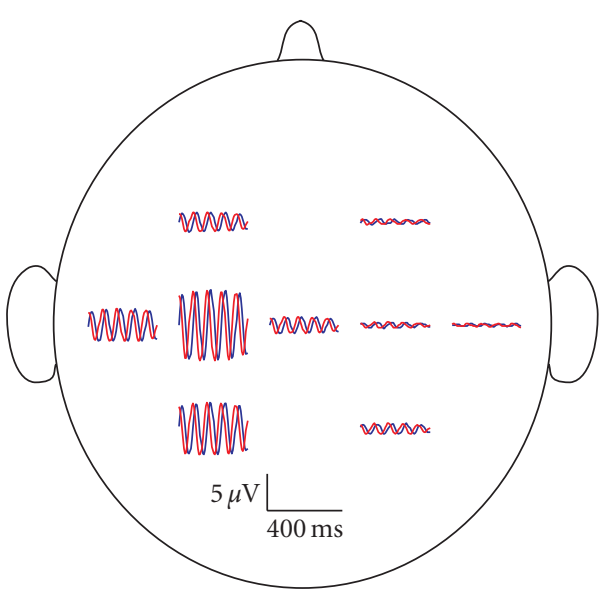

(a)

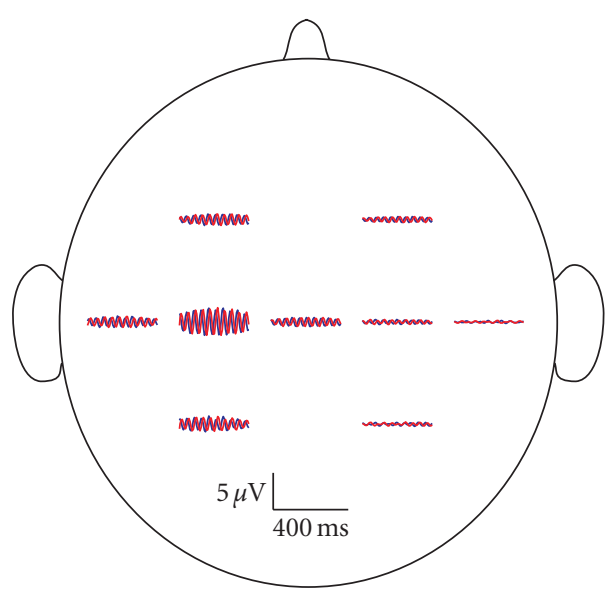

(b)

FIGURE 3: Representative waveform topographies of the four most prominent patterns for the synchronized condition (high SMR-band amplitude). The waveforms are presented with respect to the electrode locations given in Figure 2. In the left topography, the first and second most prominent patterns are illustrated in red and blue, respectively, and represent the $\mu$ frequency band. In the right topography, the third and fourth most prominent patterns are illustrated in red and blue, respectively, and represent the $\beta$ frequency band. For this user (User D), the $\mu$ band from channel $\mathrm{C}_{3}$ was used for online control.

the prominent components primarily ranged from apparent random noise to visual $\alpha$-type activity depending on the user, although other nonvisual rhythmic components were present in some users. Although the method is capable of extracting potential phase shifts between channels for a particular CSTP component, there were no apparent phase offsets between channels for any of the prominent components.

4.2. Independent Spatio-Temporal Patterns. Although the prominent CSTP components are essentially orthogonal Fourier components at the $\mu$ and $\beta$ frequencies, these components are not necessarily independent. In order to gain insight regarding the independent patterns generated for each target condition, independent component analysis (ICA) can be performed on the projected features for each condition. Because the resulting ICA unmixing matrix indicates the relative contribution of each projection, and each CSTP component corresponds to a particular projection, the ICA mixing matrix (inverse of the unmixing matrix) can be applied to the CSTP components to produce a representation of the independent patterns. These independent patterns should provide an indication of the preferred response morphologies (i.e., amplitude and phase relationships) for the different conditions. The independent common spatiotemporal patterns (ISTPs) for each target condition $i \in$ $\{1,2\}$ are attained as follows:

$$
\operatorname{CSTP}_{i}^{\mathrm{ICA}}=\left[W^{-1}\right]_{i} A_{i}
$$

where $A$ is the resultant mixing matrix of the ICA.

Figure 4 shows, for three representative users, the ISTPs of the synchronized condition that result in the highest correlation with target position. The mixing matrices were derived from the 8 most prominent projections (empirically found to be a suitable number of projections for ICA) for each target condition using FastICA [19] with a tansigmoidal nonlinearity. The classical arch-shape patterns depicted in Figure 4 are consistent for the majority of users, which supports the notion of preferred phase coupling between the $\mu$ and $\beta$ bands [4].

To confirm that the ISTPs are in fact useful and valid results, the two ISTP projections from each class (four features) having the highest correlations with the target position were extracted, and regression weights were determined using the first session from each user. The $r^{2}$ correlations of the resulting regressed feature were computed using the three subsequent sessions for each user. These $r^{2}$ values were compared to values produced by the incumbent online method using two large Laplacian channels, the online control channel, and the contralateral channel over the opposite hemisphere (see Figure 2). For each channel, $3-\mathrm{Hz} \mu$ and $\beta$ band spectral bins were extracted using a 16th-order AR model derived via the maximum entropy method (MEM) [20]. Regression weights were determined for these four features using the first session from each user, and the resulting regressed feature was correlated with three subsequent sessions for each user.

Figure 5 illustrates the $r^{2}$ for each method averaged across users. These results indicate that the specific spatiotemporal relationships of ISTP provide an improved model compared to the Laplacian and AR combination, which applies fixed spatial weights and does not utilize phase relationships.

\section{Discussion}

The prominence of the sinusoidal $\mu$ and $\beta$ components in the CSTPs reaffirms the efficacy of conventional spectral methods for a SMRs-based BCI. ISTP additionally 


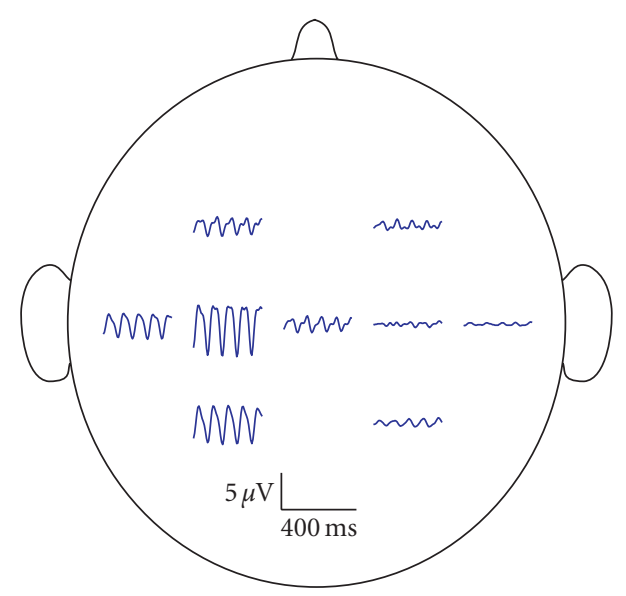

(a)

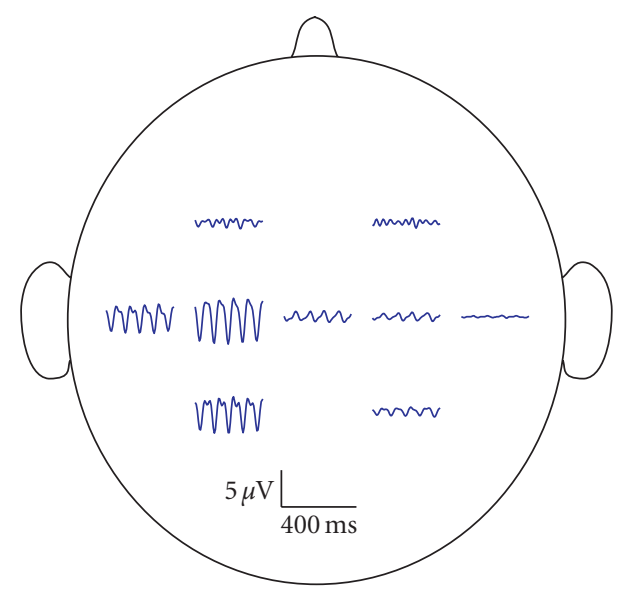

(b)

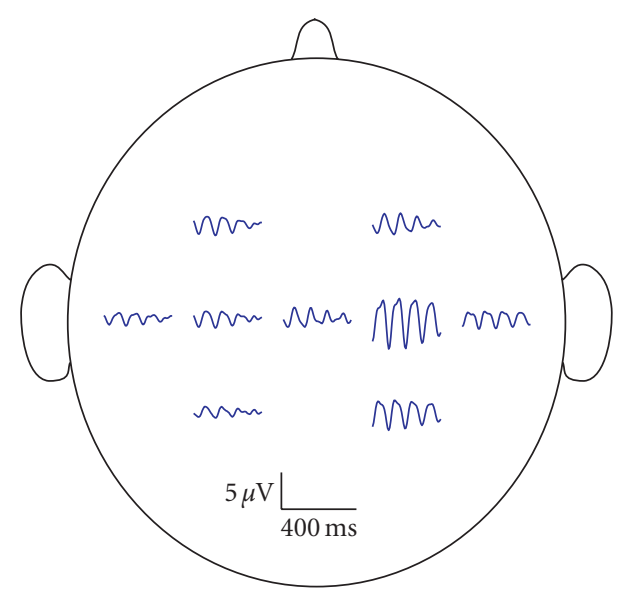

(c)

FIgURE 4: Waveform topographies of the independent common spatio-temporal patterns having the highest correlation for the synchronized condition for three representative users. The waveforms are presented with respect to the electrode locations given in Figure 2. The users depicted in the top two topographies (User $A$ and $D$, resp.) used channel $C_{3}$ for online control, while the user depicted in the bottom topography (User G) used channel $\mathrm{C}_{4}$.

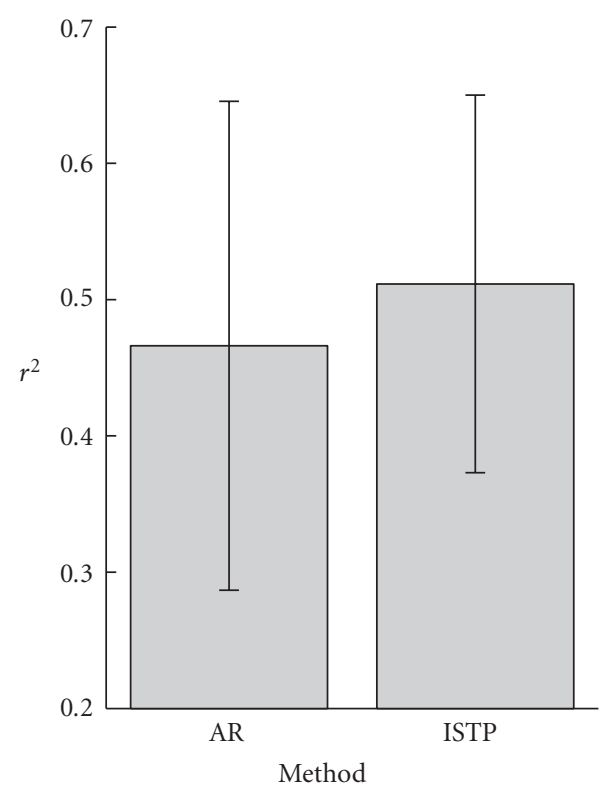

Figure 5: An offline evaluation of $r^{2}$ for the AR and ISTP methods averaged over 10 users. The error bars indicate standard deviation.

distinguishes the actual independent spatio-temporal morphologies indicative of the different mental states without prior knowledge of the SMR characteristics. As with any application of ICA where exact number of sources is not known, it is difficult to speculate about the significance of the resulting ISTPs. Nevertheless, it is interesting that the ISTPs having the highest correlation with the target position often exhibit the classical arch-shaped $\mu$-rhythm pattern [20], suggesting that there is a preferred amplitude/phase synchronization between the fundamental SMR frequency and the harmonics.

The resulting ISTP morphologies capture these preferred amplitude and phase relationships across and within channels, which can be visualized, characterized, and applied for classification purposes. The prominent ISTP patterns could potentially be used as spatio-temporal matched filter templates, which would minimize the phase effects and produce better instantaneous tracking estimates than a fixed classifier for continuous SMR cursor control [4]. However, the feature space required for ISTP would likely be overkill compared to methods such CSSSP [21] for discrete classification purposes.

In addition to machine learning techniques, a significant portion of EEG analysis and interpretation is still based on visual inspection of the signals for BCI and clinical applications. The true utility of ISTP is visualizing the complete spatio-temporal morphologies associated with particular mental states, which is not possible with CSSSP. Accordingly, the method of ISTP can potentially be used for visualizing and characterizing lesser known or more complex userspecific EEG responses such as steady-state visual evoked potentials (SSVEPs). By using noncentered covariance matrices as suggested in [22], the CSTP method can be applied to transient responses such as P300 event-related potentials [23]. This modification can also potentially be used to 
concurrently capture class-dependent components due to slow cortical potentials, movement related potentials, and SMRs for tasks where all such responses are relevant.

\section{Acknowledgments}

This work was initiated and partially completed at the Wadsworth Center, NYSDOH, Albany, NY and supported in part by the National Institutes of Health under Grant NICHD HD30146 and Grant NIBIB/NINDS EB00856 and in part by the James S. McDonnell Foundation. The author would also like to thank Dennis J. McFarland and Jonathan R. Wolpaw for their valuable input to the manuscript.

\section{References}

[1] S. Lemm, B. Blankertz, G. Curio, and K.-R. Müller, "Spatiospectral filters for improving the classification of single trial EEG," IEEE Transactions on Biomedical Engineering, vol. 52, no. 9, pp. 1541-1548, 2005.

[2] E. Niedermeyer and F. L. da Silva, Eds., Electroencephalography: Basic Principles, Clinical Applications and Related Fields, Williams and Wilkins, Baltimore, Md, USA, 5th edition, 2004.

[3] M. Cheng, W. Jia, X. Gao, S. Gao, and F. Yang, "Mu rhythm-based cursor control: an offline analysis," Clinical Neurophysiology, vol. 115, no. 4, pp. 745-751, 2004.

[4] D. J. Krusienski, G. Schalk, D. J. McFarland, and J. R. Wolpaw, "A $\mu$-rhythm matched filter for continuous control of a brain-computer interface," IEEE Transactions on Biomedical Engineering, vol. 54, no. 2, pp. 273-280, 2007.

[5] K. Fukunaga, Introduction to Statistical Pattern Recognition, Academic Press, San Diego, Calif, USA, 2nd edition, 1990.

[6] Z. J. Koles and A. C. K. Soong, "EEG source localization: implementing the spatio-temporal decomposition approach," Electroencephalography and Clinical Neurophysiology, vol. 107, no. 5, pp. 343-352, 1998.

[7] G. Dornhege, B. Blankertz, G. Curio, and K.-R. Müller, "Boosting bit rates in noninvasive EEG single-trial classifications by feature combination and multiclass paradigms," IEEE Transactions on Biomedical Engineering, vol. 51, no. 6, pp. 9931002, 2004.

[8] J. Müller-Gerking, G. Pfurtscheller, and H. Flyvbjerg, "Designing optimal spatial filters for single-trial EEG classification in a movement task," Clinical Neurophysiology, vol. 110, no. 5, pp. 787-798, 1999.

[9] P. Shenoy, M. Krauledat, B. Blankertz, R. P. N. Rao, and K.R. Müller, "Towards adaptive classification for BCI," Journal of Neural Engineering, vol. 3, no. 1, pp. R13-R23, 2006.

[10] G. Townsend, B. Graimann, and G. Pfurtscheller, "A comparison of common spatial patterns with complex band power features in a four-class BCI experiment," IEEE Transactions on Biomedical Engineering, vol. 53, no. 4, pp. 642-651, 2006.

[11] Y. Wang, P. Berg, and M. Scherg, "Common spatial subspace decomposition applied to analysis of brain responses under multiple task conditions: a simulation study," Clinical Neurophysiology, vol. 110, no. 4, pp. 604-614, 1999.

[12] D. J. McFarland, L. M. McCane, S. V. David, and J. R. Wolpaw, "Spatial filter selection for EEG-based communication," Electroencephalography and Clinical Neurophysiology, vol. 103, no. 3, pp. 386-394, 1997.

[13] D. J. McFarland, W. A. Sarnacki, and J. R. Wolpaw, "Braincomputer interface (BCI) operation: optimizing information transfer rates," Biological Psychology, vol. 63, no. 3, pp. 237251, 2003.

[14] D. J. McFarland and J. R. Wolpaw, "EEG-based communication and control: speed-accuracy relationships," Applied Psychophysiology Biofeedback, vol. 28, no. 3, pp. 217-231, 2003.

[15] J. R. Wolpaw and D. J. McFarland, "Multichannel EEG-based brain-computer communication," Electroencephalography and Clinical Neurophysiology, vol. 90, no. 6, pp. 444-449, 1994.

[16] J. R. Wolpaw and D. J. McFarland, "Control of a twodimensional movement signal by a noninvasive braincomputer interface in humans," Proceedings of the National Academy of Sciences of the United States of America, vol. 101, no. 51, pp. 17849-17854, 2004.

[17] G. Schalk, D. J. McFarland, T. Hinterberger, N. Birbaumer, and J. R. Wolpaw, "BCI2000: a general-purpose brain-computer interface (BCI) system," IEEE Transactions on Biomedical Engineering, vol. 51, no. 6, pp. 1034-1043, 2004.

[18] F. Sharbrough, C. E. Chatrian, R. P. Lesser, H. Luders, M. Nuwer, and T. W. Picton, "American electroencphalographic society guidelines for standard electrode position nomenclature," Journal of Clinical Neurophysiology, vol. 8, no. 2, pp. 200202, 1991.

[19] A. Hyvärinen, "Fast and robust fixed-point algorithms for independent component analysis," IEEE Transactions on Neural Networks, vol. 10, no. 3, pp. 626-634, 1999.

[20] W. N. Kuhlman, "EEG feedback training of epileptic patients: clinical and electroencephalographic analysis," Electroencephalography and Clinical Neurophysiology, vol. 45, no. 6, pp. 699-710, 1978.

[21] G. Dornhege, B. Blankertz, M. Krauledat, F. Losch, G. Curio, and K.-R. Müller, "Combined optimization of spatial and temporal filters for improving brain-computer interfacing," IEEE Transactions on Biomedical Engineering, vol. 53, no. 11, pp. 2274-2281, 2006.

[22] G. Dornhege, B. Blankertz, and G. Curio, "Speeding up classification of multi-channel brain-computer interfaces: common spatial patterns for slow cortical potentials," in Proceedings of the 1st International IEEE EMBS Conference on Neural Engineering, pp. 591-594, 2003.

[23] D. J. Krusienski, E. W. Sellers, and T. M. Vaughan, "Common spatio-temporal patterns for the P300 speller," in Proceedings of the 3rd International IEEE EMBS Conference on Neural Engineering, pp. 421-424, 2007. 